\title{
On a Method of Carasso and Laurent for Constructing Interpolating Splines
}

\author{
By M. J. Munteanu and L. L. Schumaker*
}

\begin{abstract}
Carasso and Laurent studied a method for computing natural polynomial splines interpolating simple data. We discuss several similar methods which can be applied to numerical construction of more general interpolating splines, including $L g$-splines interpolating Extended-Hermite-Birkhoff data.
\end{abstract}

1. Introduction. Let $x_{0}<x_{1}<\cdots<x_{N+1}$. A natural polynomial spline of degree $2 m-1$ with knots at the $\left\{x_{i}\right\}_{0}^{N+1}$ and interpolating data $\left\{y_{i}\right\}_{0}^{N+1}$ at the knots is a function $s$ with the properties

(1.1) $s$ is a polynomial of degree $2 m-1$ in $\left(x_{i}, x_{i+1}\right), \quad i=0,1, \cdots, N$;

(1.2) (interpolation) $\quad s\left(x_{i}\right)=y_{i}, \quad i=0,1, \cdots, N+1$;

(1.3) (smoothness) $\quad s^{(i)}\left(x_{i}+\right)=s^{(j)}\left(x_{i}-\right)$,

$$
j=0,1, \cdots, 2 m-2 ; i=1,2, \cdots, N ;
$$

and

(1.4) (end conditions) $s^{(m)}\left(x_{0}+\right)=\cdots=s^{(2 m-2)}\left(x_{0}+\right)=0$, $s^{(m)}\left(x_{N+1}-\right)=\cdots=s^{(2 m-2)}\left(x_{N+1}-\right)=0$.

There are a wide variety of computational schemes for numerical construction of such splines (see e.g. [1], [3], [5], [6], [9], [10], [11], [12], [15] and the references therein). Carasso and Laurent [3] proposed a certain factorization method which proceeds roughly as follows. Let $\bar{s}(x)=\left[s(x), \cdots, s^{(2 m-1)}(x)\right]^{T}$ and $R_{i}=\bar{s}\left(x_{i}+\right)$, $L_{i}=\tilde{s}\left(x_{i}-\right)$. Clearly, (1.2) and (1.4) provide $m$ relations on the components of $R_{0}$. But, in view of (1.1), it is easy to obtain (using the Taylor expansion) $m$ relations on the components of $L_{1}$ from those on $R_{0}$. Furthermore, the smoothness property (1.3) at $x_{1}$ and the interpolation condition permit the derivation of $m$ relations on $R_{1}$ from those on $L_{1}$. Continuing this process, $m$ relations can be found on all of the vectors $\left\{L_{i}\right\}_{1}^{N+1}$ and $\left\{R_{i}\right\}_{0}^{N}$. Similarly, starting with $L_{N+1}$ and proceeding backwards, a separate set of $m$ relations on each of these vectors can be derived. Finally, for each $i$, the sets of relations can be combined to obtain $2 m$ equations for the $2 m$ components of the $L_{i}$ and $R_{i}$, which, of course, determines the spline in each interval.

The purpose of this paper is to discuss such factorization schemes in a general framework and to derive several explicit methods which can be utilized for the numer-

Received April 2, 1972.

AMS (MOS) subject classifications (1970). Primary 65D05; Secondary 41A15.

* Supported in part by AFOSR-69-1812-C.

Copyright (c) 1973, American Mathematical Society 
ical construction of considerably more general interpolating splines, including, for example, $L g$-splines interpolating Extended-Hermite-Birkhoff data.

2. Preliminaries. The basic structural properties underlying the factorization method outlined in Section 1 for polynomial splines are shared by a wide class of generalizations (e.g. $g$-splines, $L$-splines, and $L g$-splines; see Section 4). It is convenient to abstract these properties.

Let $x_{0}<x_{1}<\cdots<x_{N+1}$, and let $\left\{u_{i}\right\}_{0}^{2 m-1}$ be $2 m$ functions such that the Wronskian $W\left(u_{0}, \cdots, u_{2 m-1}\right)(x)>0$ for $x \in\left[x_{0}, x_{N+1}\right]$. We consider construction of a spline function $s(x)$ with the following properties (as before, we use the notation $\bar{s}(x)=$ $\left[s(x), \cdots, s^{(2 m-1)}(x)\right]^{T}$ and $\left.L_{i}=\bar{s}\left(x_{i}-\right), R_{i}=\bar{s}\left(x_{i}+\right)\right)$ :

(2.1) (piecewise property). $s$ is a linear combination of $\left\{u_{i}\right\}_{0}^{2 m-1}$ in each interval $\left(x_{i}, x_{i+1}\right), i=0,1, \cdots, N$.

(2.2) (interpolating conditions). For each $i=1,2, \cdots, N$, there exist $1 \leqq z_{i} \leqq m$, a $z_{i}$-vector $\gamma_{i}$, and a $z_{i} \times 2 m\left(\operatorname{rank} z_{i}\right)$ matrix $A_{i}$ such that $A_{i} \bar{s}\left(x_{i}\right)=\gamma_{i}$.

(2.3) (smoothness conditions). For each $i=1,2, \cdots, N$, there exists a $\left(2 m-z_{i}\right)$ $\times 2 m$ matrix $S_{i}$ of rank $2 m-z_{i}$ such that $S_{i} L_{i}=S_{i} R_{i}$.

(2.4) (end conditions). There exist $m \times 2 m$ rank $m$ matrices $R F_{0}$ and $L B_{N+1}$ and $m$-vectors $r_{0}$ and $l_{N+1}^{\prime}$ such that $R F_{0} R_{0}=r_{0}, L B_{N+1} L_{N+1}=l_{N+1}^{\prime}$.

We need the following elementary fact.

LEMMA 2.1. Given a real number $y$, suppose $s(x)$ is a linear combination of $\left\{u_{j}(x)\right\}_{0}^{2 m-1}$ in some interval $[\alpha, \beta]$ containing $y$. Then, there exists a set of functions $\left\{u_{i}(y ; x)\right\}_{0}^{2 m-1}$ in $U=\operatorname{span}\left\{u_{j}(x)\right\}_{0}^{2 m-1}$ such that, for every $x \in[\alpha, \beta]$,

$$
s(x)=\sum_{i=0}^{2 m-1} s^{(i)}(y) u_{j}(y ; x) .
$$

Proof. By the Wronskian assumption on the functions of $U$, the linear functionals $\left\{\lambda_{i}\right\}_{0}^{2 m-1}$ given by $\lambda_{j} f=f^{(j)}(y)$ are linearly independent in the dual $U^{*}$. Theorem 2.5.1 of Davis [4] applies.

The functions $u_{j}(y ; x)$ in the above lemma depend only on $U$ and $y$, and not on $\alpha, \beta$. The following immediate consequence of the lemma shows that, in each interval $\left(x_{i}, x_{i+1}\right)$, a spline $s$ is determined by either $R_{i}$ or $L_{i+1}$.

COROLlary 2.2. For $k=0,1, \cdots, N+1$, let $\left\{u_{j}\left(x_{k} ; x\right)\right\}_{0}^{2 m-1}$ be the set of functions corresponding to $y=x_{k}$ in Lemma 2.1. Suppose s satisfies (2.1). Then

$$
\begin{aligned}
& \bar{s}(x)=W\left(u_{0}\left(x_{i} ; \cdot\right), \cdots, u_{2 m-1}\left(x_{i} ; \cdot\right)\right)(x) R_{i}, \\
& s(x)=W\left(u_{0}\left(x_{i+1} ; \cdot\right), \cdots, u_{2 m-1}\left(x_{i+1} ; \cdot\right)\right)(x) L_{i+1},
\end{aligned}
$$

for $x \in\left(x_{i}, x_{i+1}\right), i=0,1, \cdots, N$.

COROLLARY 2.3. Let $s$ satisfy (2.1). Then, there exist $2 m \times 2 m$ nonsingular matrices $T F_{i}$ and $T B_{i}$ such that

$$
\begin{aligned}
L_{i+1} & =T F_{i} R_{i}, \\
R_{i} & =T B_{i} L_{i+1} .
\end{aligned}
$$

Proof. For example, from Corollary 2.2, we see that

$$
T F_{i}=W\left(u_{0}\left(x_{i} ; \cdot\right), \cdots, u_{2 m-1}\left(x_{i} ; \cdot\right)\right)\left(x_{i+1}\right) .
$$


As we remarked above, to construct a spline $s$ satisfying (2.1)-(2.4) it suffices to find either $L_{i}$ or $R_{i}$ for each $i=0,1, \cdots, N+1$. To this end, we now seek to find $2 m$ conditions on the components of each of the $L_{i}$ and $R_{i}$.

First, starting with the end condition $R F_{0} R_{0}=r_{0}$, we show how to find $m$ conditions on each of the $L_{i}$ and $R_{i}$. If we substitute $R_{0}=T B_{0} L_{1}$ in $R F_{0} R_{0}=r_{0}$, we get $L F_{1} L_{1}=l_{1}$ with $L F_{1}=R F_{0} T B_{0}$ and $l_{1}=r_{0}$ (since $R F_{0}$ is $m \times 2 m$ so is $L F_{1}$, and these are the desired $m$ relations on $L_{1}$ ). To find conditions on $R_{1}$, consider

$$
\left[\begin{array}{c|c}
L F_{i} & 0 \\
\hline S_{i} & -S_{i}
\end{array}\right]\left[\begin{array}{c}
L_{i} \\
R_{i}
\end{array}\right]=\left[\begin{array}{l}
l_{i} \\
0
\end{array}\right]
$$

with $i=1$. The matrix

$$
\left[\begin{array}{c}
L F_{i} \\
S_{i}
\end{array}\right]
$$

is $3 m-z_{i}$ by $2 m$. Suppose the rows of $L F_{i}$ are rearranged so that the last $e_{i}$ rows coupled with the $2 m-z_{i}$ rows of $S_{i}$ are linearly independent. Since $S_{i}$ is of rank $2 m-z_{i}, 0 \leqq e_{i} \leqq z_{i}$. Now, using the $2 m-z_{i}$ rows of the matrix $S_{i}$ and the last $e_{i}$ rows from $L F_{i}$, we can eliminate the first $2 m$ variables from the first $m-z_{i}$ of the equations. This leaves $m-z_{i}$ equations on the components of $R_{i}$. Adding the $z_{i}$ equations $A_{i} R_{i}=\gamma_{i}$, we have $m$ equations on $R_{i}$ which can be written $R F_{i} R_{i}=r_{i}$. These steps can be repeated to go from $m$ conditions on $R_{1}$ to $m$ conditions on $L_{2}$, then to $R_{2}$, and so on. We conclude there exist $m \times 2 m$ matrices $L F_{i}, R F_{i}$ and $m$ vectors $l_{i}, r_{i}$ such that

$$
\begin{aligned}
L F_{i} L_{i}=l_{i}, & i=1,2, \cdots, N+1, \\
R F_{i} R_{i}=r_{i}, & i=0,1, \cdots, N .
\end{aligned}
$$

In general, $L F_{i}=R F_{i-1} T B_{i-1}$ and $l_{i}=r_{i-1}, i=1,2, \cdots, N+1$.

Conditions (2.11) and (2.12) were constructed by starting with the left end condition and proceeding forward (the $F$ in $R F$ is to remind us of that). A similar procedure starting with $L B_{N+1} L_{N+1}=l_{N+1}^{\prime}$ and proceeding backwards produces $m \times 2 m$ matrices $L B_{i}, R B_{i}$ and $m$-vectors $l_{i}^{\prime}, r_{i}^{\prime}$ such that

$$
\begin{array}{ll}
L B_{i} L_{i}=l_{i}^{\prime}, & i=1,2, \cdots, N+1, \\
R B_{i} R_{i}=r_{i}^{\prime}, & i=0,1, \cdots, N .
\end{array}
$$

Here, $R B_{i}=L B_{i+1} T F_{i}$ and $r_{i}^{\prime}=l_{i+1}^{\prime}$. The matrix $L B_{i}$ is found from $R B_{i}$ by eliminating the last $2 m$ variables from the first $m-z_{i}$ rows of

$$
\left[\begin{array}{c|c}
0 & R B_{i} \\
\hline S_{i} & -S_{i}
\end{array}\right]\left[\begin{array}{c}
L_{i} \\
R_{i}
\end{array}\right]=\left(\begin{array}{c}
r_{i}^{\prime} \\
0
\end{array}\right)
$$

and adding the relations $A_{i} L_{i}=\gamma_{i}$. We assume for the remainder of the paper that if $S_{i} L_{i}=S_{i} R_{i}$, then $A_{i} R_{i}=\gamma_{i}$, if and only if $A_{i} L_{i}=\gamma_{i}$. The following lemma will be needed later.

LemMA 2.4. Suppose $R_{i}^{*}$ is a vector satisfying (2.12) and (2.14). Then there exists 
a vector $L_{i}^{*}$ satisfying (2.11) and (2.13). Conversely, given $L_{i}^{*}$ satisfying (2.11), (2.13) there exists $R_{i}^{*}$ satisfying (2.12), (2.14).

Proof. Let $R_{i}^{*}$ satisfy (2.12) and (2.14). We show how to construct $L_{i}^{*}$. Let $\widetilde{L F}_{i}$ be a $z_{i} \times 2 m$ matrix formed by augmenting the last $e_{i}$ rows of $L F_{i}$ (cf. the discussion following (2.10)) in such a way that the matrix in (2.16) below is nonsingular. Let $L_{i}^{*}$ be the solution of

$$
\left[\begin{array}{c}
\widetilde{L F_{i}} \\
S_{i}
\end{array}\right] L_{i}^{*}=\left[\begin{array}{c}
0 \\
\tilde{l}_{i} \\
S_{i} R_{i}^{*}
\end{array}\right]
$$

where $l_{i}$ consists of the last $e_{i}$ rows of $l_{i}$.

By the construction of (2.12), $R F_{i} R_{i}^{*}=r_{i}$ implies $A_{i} R_{i}^{*}=\gamma_{i}$. Since $S_{i} R_{i}^{*}=S_{i} L_{i}^{*}$, it follows that $A_{i} L_{i}^{*}=\gamma_{i}$. Now we claim $L F_{i} L_{i}^{*}=l_{i}$ and $L B_{i} L_{i}^{*}=l_{i}^{\prime}$. Indeed, suppose $L F_{i} L_{i}^{*} \neq l_{i}$. Then

$$
\left.\begin{array}{|c|c|}
\hline L F_{i} & 0 \\
\hline S_{i} & -S_{i}
\end{array}\right]\left[\begin{array}{c}
L_{i}^{*} \\
R_{i}^{*}
\end{array}\right]=\left[\begin{array}{c}
l_{i}+\delta_{i} \\
0
\end{array}\right]
$$

for some $\delta_{i}$. By the construction of $L_{i}^{*}$, the last $e_{i} \delta_{i}$ 's are zero. Suppose at least one of the first $m-e_{i}$ is not. Now, performing the same elimination used on (2.10) leads to a contradiction of $R F_{i} R_{i}^{*}=r_{i}$. To check that $L B_{i} L_{i}^{*}=l_{i}^{\prime}$, note that (2.15) holds for $L_{i}^{*}$ and $R_{i}^{*}$. Eliminating the last $2 m$ variables in the first $m-z_{i}$ equations and using $A_{i} L_{i}^{*}=\gamma_{i}$ yields $L B_{i} L_{i}^{*}=l_{i}^{\prime}$.

The converse assertion is proved analogously.

3. Constructive Methods. In this section, we discuss three methods for constructing a spline $s$ satisfying (2.1)-(2.4). The methods are based on computing $L_{i}$ and/or $R_{i}$ for each $i=0,1, \cdots, N+1$ from the information on $s$ inherent in the relations (2.11)-(2.14).

Method 1. For $i=1,2, \cdots, N+1$, solve

$$
\left[\begin{array}{l}
L F_{i} \\
L B_{i}
\end{array}\right] L_{i}=\left[\begin{array}{l}
l_{i} \\
l_{i}^{\prime}
\end{array}\right]
$$

Method 2. For $i=1,2, \cdots, N$, solve

$$
\left[\begin{array}{c|c}
L F_{i} & 0 \\
\hline 0 & R B_{i} \\
\hline A_{i} \mid 0 & 0 \\
\hline S_{i} & -S_{i}
\end{array}\right]\left[\begin{array}{l}
L_{i} \\
R_{i}
\end{array}\right]=\left[\begin{array}{c}
l_{i} \\
r_{i} \\
\gamma_{i} \\
0
\end{array}\right] .
$$

Method 3. Solve

$$
\left[\begin{array}{l}
L F_{N+1} \\
L B_{N+1}
\end{array}\right] L_{N+1}=\left[\begin{array}{l}
l_{N+1} \\
l_{N+1}^{\prime}
\end{array}\right]
$$


For $i=N, N-1, \cdots, 1$, find

$$
R_{i}=T B_{i} L_{i+1}
$$

and solve

$$
\left[\begin{array}{c}
\widetilde{L F}_{i} \\
S_{i}
\end{array}\right] L_{i}=\left[\begin{array}{c}
0 \\
\tilde{l}_{i} \\
S_{i} R_{i}
\end{array}\right],
$$

where $\widetilde{L F}_{i}$ and $l_{i}$ are as in the proof of Lemma 2.4 (see (2.16)).

THEOREM 3.1. Suppose there is a unique spline function s satisfying (2.1)-(2.4). Then Methods 1-3 are well defined; i.e., the systems in (3.1)-(3.3) and (3.5) are nonsingular.

Proof. We consider first Method 1. Suppose (3.1) is singular for some $k, 1 \leqq k \leqq$ $N+1$. Then, there exists $L_{k}^{*} \neq 0$ with

$$
\left[\begin{array}{l}
L F_{k} \\
L B_{k}
\end{array}\right] L_{k}^{*}=0 .
$$

For $x \in\left(x_{k-1}, x_{k}\right)$ define

$$
s^{*}(x)=\sum_{j=0}^{2 m-1} L_{k}^{*}(j) u_{j}\left(x_{k} ; x\right) .
$$

Clearly, $R_{k-1}^{*}=\bar{s}^{*}\left(x_{k-1}+\right)$ satisfies $R F_{k-1} R_{k-1}^{*}=R B_{k-1} R_{k-1}^{*}=0$. Now, by Lemma 2.4 , we can find $L_{k-1}^{*}$ satisfying

$$
\left[\begin{array}{l}
L F_{k-1} \\
L B_{k-1}
\end{array}\right] L_{k-1}^{*}=0 .
$$

This process can be continued to define $s^{*}(x)$ in $\left[x_{0}, x_{k}\right]$. A similar process starting with $L_{k}^{*}$ and proceeding forward can be used to define $s^{*}(x)$ throughout $\left[x_{0}, x_{N+1}\right]$ with the property that (2.11)-(2.14) all hold with zero right-hand sides. Since

$$
\left[\begin{array}{l}
L F_{i} \\
L B_{i}
\end{array}\right] L_{i}^{*}=0
$$

implies $A_{i}=0$, we conclude that $s^{*}$ is a nontrivial spline satisfying (2.1)-(2.4) with homogeneous data. This contradicts the uniqueness assumption.

For Method 2, suppose (3.2) is singular. Then, for some $k, 1 \leqq k \leqq N$, there exist nontrivial $L_{k}^{*}$ and $R_{k}^{*}$ with

$$
\left[\begin{array}{c|c}
L F_{k} & 0 \\
\hline 0 & R B_{k} \\
\hline A_{k} \mid 0 & 0 \\
\hline S_{k} & -S_{k}
\end{array}\right]\left[\begin{array}{c}
L_{k}^{*} \\
R_{k}^{*}
\end{array}\right]=0 .
$$


Moreover, performing elimination on (3.6), we conclude that $R F_{k} R_{k}^{*}=L B_{k} L_{k}^{*}=0$. Now, $s^{*}(x)$ can be defined in $\left[x_{k-1}, x_{k}\right]$ and $\left[x_{k}, x_{k+1}\right]$ in terms of $L_{k}^{*}$ and $R_{k}^{*}$, respectively. Lemma 2.4 can be used to extend $s^{*}$ to $\left[x_{0}, x_{N+1}\right]$ such that $(2.11)-(2.14)$ hold with 0 right-hand sides. This is again a contradiction of the uniqueness of spline interpolation, as $s^{*} \not \equiv 0$.

Finally, for Method 3, we note that the system (3.3) cannot be singular (by the same proof as for Method 1). The matrix (3.5) is nonsingular by construction (cf. the proof of Lemma 2.4).

The matrix in (3.5) was obtained from $L F_{i}$ and $S_{i}$ by an augmentation process. In view of the uniqueness of the interpolating spline, we see that the solution of (3.5) must be unique, and so any augmentation must give the same solution. But this means that there is no freedom to choose different augmentations and we conclude that $e_{i}=z_{i}$ in the proofs of Corollary 2.3 and Lemma 2.4. Thus no augmentation is ever necessary in practice.

4. Applications. We recall the definition of $L g$-splines [8]. Let $L$ be an $m$ th order differential operator, $\Lambda=\left\{\lambda_{i}\right\}_{1}^{n}$ a set of linearly independent linear functionals on $H_{2}^{m}$, and $\left\{d_{j}\right\}_{1}^{n}$ a set of real numbers. An $L g$-spline interpolating $\left\{d_{i}\right\}_{1}^{n}$ with respect to $\Lambda$ is a solution of

$$
\begin{aligned}
\|L s\|_{2} & =\min _{f \in U(d)}\|L f\|_{2}, \\
U(d) & =\left\{f \in H_{2}^{m}: \lambda_{j} f=d_{i}, j=1,2, \cdots, n\right\} .
\end{aligned}
$$

The methods of Section 3 can be used to construct such splines when $\Lambda$ corresponds to EHB data.

To describe EHB interpolation, let $x_{0}<x_{1}<\cdots<x_{N+1}$ and let $1 \leqq z_{i} \leqq m$ with $\sum_{i=0}^{N+1} z_{i}=n$. For $0 \leqq i \leqq N+1,1 \leqq j \leqq z_{i}$, let $\alpha(i, j)=\left(\alpha_{0}(i, j), \cdots, \alpha_{m-1}(i, j)\right)$ be vectors such that for each $i$ the vectors in the set $\{\alpha(i, j)\}_{j=1}^{z i}$ are linearly independent. $\Lambda$ generates an EHB interpolation problem if the $n$ linear functionals in $\Lambda$ are

$$
\sum_{k=0}^{m-1} \alpha_{k}(i, j) D_{x_{i}}^{k}, \quad j=1,2, \cdots, z_{i} ; i=0,1, \cdots, N+1,
$$

where $D_{x_{i}}^{k} f=f^{(k)}\left(x_{i}\right)$.

We now show that an $L g$-spline $s$ interpolating EHB data satisfies the conditions (2.1)-(2.4). At each $x_{i}$, there are $z_{i}$ interpolating conditions which can be written in the form of (2.2) with

$$
A_{i}=\left[\begin{array}{c|c}
\alpha(i, 1) & \\
\vdots & 0 \\
\alpha\left(i, z_{i}\right) &
\end{array}\right], \quad i=1,2, \cdots, N
$$

Furthermore (see [8, Theorem 3.6]), $s$ belongs to $C^{m-1}$ and at each $x_{i}, 1 \leqq i \leqq N$, there are additional $m-z_{i}$ smoothness conditions. These are given by $R_{i}^{\left(x_{i}\right)} s\left(x_{i}+\right)=$ $R_{i}^{\left(x_{i}\right)} s\left(x_{i}-\right), j=z_{i}+1, \cdots, m$, where $R_{i}^{\left(x_{i}\right)} s(x)=\sum_{k=m}^{2 m-1} \beta_{k}(i, j) s^{(k)}(x)$, with certain (real) coefficients $\beta_{k}(i, j)$. Thus, the smoothing matrices of (2.3) are 


$$
S_{i}=\left[\begin{array}{c|c}
I_{m} & 0 \\
\hline 0 & \beta\left(i, z_{i}+1\right) \\
& \vdots \\
& \beta(i, m)
\end{array}\right] .
$$

Finally, the end conditions on $s$ are given by (2.4) with

$$
R F_{0}=\left[\begin{array}{c|c}
\alpha(0,1) & \\
\vdots & \alpha\left(0, z_{0}\right) \\
& \beta\left(0, z_{0}+1\right) \\
0 & \vdots \\
& \beta(0, m)
\end{array}\right], \quad r_{0}=\left[\begin{array}{c}
d_{1} \\
\vdots \\
d_{z_{0}} \\
0 \\
\vdots \\
0
\end{array}\right]
$$

and

$$
L F_{N+1}=\left[\begin{array}{c|c}
\alpha(N+1,1) & \\
\vdots & 0 \\
0 & \beta\left(N+1, z_{N+1}\right) \\
\hline & \beta(N+1, m)
\end{array}\right], \quad l_{N+1}^{\prime}=\left[\begin{array}{c}
d_{n-z+1}+1 \\
\vdots \\
d_{n} \\
0
\end{array}\right] .
$$

For the smoothness matrices of (4.3), the condition $S_{i} L_{i}=S_{i} R_{i}$ clearly implies the first $m$ components of $L_{i}$ and $R_{i}$ are the same. Thus, the hypothesis needed for Lemma 2.4 that $A_{i} L_{i}=\gamma_{i}$ iff $A_{i} R_{i}=\gamma_{i}$ holds for these splines.

The matrices $A_{i}, S_{i}, R F_{0}$, and $L F_{N+1}$ are considerably simplified for less general forms of data. For example, for HB data (see [8]) each of the vectors $\alpha(i, j)$ and $\beta(i, k)$ has only one nonzero coefficient. For Hermite data,

$$
A_{i}=\left[I_{z i} \mid 0\right], \quad S_{i}=\left[\begin{array}{c|c}
I_{m} & 0 \\
\hline 0 & I_{m-z i}
\end{array}\right]
$$

and

$$
R F_{0}=\left[\begin{array}{c|c}
I_{z_{0}} & 0 \\
\hline 0 & I_{m-z_{0}}
\end{array}\right], \quad L F_{N+1}=\left[\begin{array}{c|c}
I_{z_{N+1}} & 0 \\
\hline 0 & I_{m-z_{N+1}}
\end{array}\right]
$$

When $L=D^{m}$, the $L g$-splines are just polynomial splines. In this case, $u_{j}(x)=x^{i}$, $j=0,1, \cdots, 2 m-1$, and the functions of Corollary 2.2 are $u_{i}\left(x_{i} ; x\right)=\left(x-x_{i}\right)^{i}$, $j=0,1, \cdots, 2 m-1$. 
5. Remarks. 1. If Method 1 is applied to the computation of a natural polynomial spline interpolating simple data $\left(z_{0}=z_{1}=\cdots=z_{N+1}=1\right)$, we have the method introduced by Carasso and Laurent [3]. Method 2 was introduced for type I $L$-splines by Munteanu [12].

2. There are a variety of methods using bases for computing polynomial interpolating splines with Hermite data (cf. the discussions in [1], [3], [5], [6], [8], [9], [11], [15]). These fall into two classes: methods where bases for the space of splines $\delta$ are used and those where bases for the space $L S$ are used (called projection methods). For polynomial splines with Hermite data, convenient local-support bases have been found ([5], [7], [11]). Similar bases have been found for $g$-splines with Hermite-Birkhoff data [9], but to date not for EHB data or for more general operators $L$. The projective methods [1], [3], [6] can be used for $L$-splines with Hermite data [12]. (Projective methods have the drawback that bases (even local support bases) for $L S$ lead to nonlocal bases for $S$, with attendant conditioning problems.)

3. Methods for computing $L g$-splines have a variety of practical applications; e.g. in constructing optimal quadrature rules (see e.g. Karlin [10, p. 83] and references therein) and solving two-point-boundary-value problems [2], [6]. An optimal method for boundary value problems of Golomb [6] requires the construction of $L g$-spline corresponding to EHB data.

4. There are some obvious dual versions of Methods 1 and 3. Method $1^{\prime}$ would involve solving

$$
\left[\begin{array}{l}
R F_{i} \\
R B_{i}
\end{array}\right] R_{i}=\left[\begin{array}{l}
r_{i} \\
r_{i}^{\prime}
\end{array}\right], \quad i=0,1, \cdots, N .
$$

A Method $3^{\prime}$ can be constructed by starting with $x_{0}$; we compute $R_{0}$ and proceed forward. It should be cautioned that the natural dual to Method 2 (that is, the use of $R F_{i}$ and $L B_{i}$ to determine $L_{i}$ and $R_{i}$ ) would not be well defined. The reason is that both sets of $m$ relations are obtained by adding the interpolating conditions to other sets of $m-z_{i}$ relations.

5. The methods of Section 3 apply equally well to type III $L$-splines [14]. The periodic case (type IV) is not covered. It may be possible to develop factorization methods for the case of splines satisfying inequalities (see [8]) rather than interpolating data exactly, and for smoothing splines (see [11]).

The methods of Section 3 apply equally well to type III $L$-splines [14]. The periodic case (type IV) is not covered. It may be possible to develop factorization methods for the case of splines satisfying inequalities (see [8]) rather than interpolating data exactly, and for smoothing splines (see [11]).

6. The methods of Section 3 can be compared with each other and with other methods in the literature for efficiency. Without examining operation counts, it is clear that Method 3 requires only half the effort to set up that the other two do as the $\left\{L F_{i}\right\}_{1}^{N}$ and $\left\{R B_{i}\right\}_{1}^{N}$ are not needed. The actual operation counts for finding $\left\{L F_{i}\right\}_{1}^{N+1}$ depend on the complexity of the interpolation and smoothing conditions. For polynomial splines, the total operation count for any of the algorithms is of the order con $\mathrm{Nm}^{3}$, where con does not depend on $N$ or $m$ (but does depend on the algorithm). The storage required for the factorization method is of order $\mathrm{Nm}^{2}$. Local support basis methods use on the order of $\mathrm{Nm}$ storage if the band structure is accounted for, and $N^{2}$ otherwise. 
7. Method 3 has been tested for a wide variety of simple, Hermite, and HermiteBirkhoff interpolation problems for polynomial splines. For complete FORTRAN subroutines and a discussion of the numerical experience with them, see [Eidson, H. L. and Schumaker, L. L., Computation of g-Splines via a Factorization Method, CNA Report 60, Center for Numerical Analysis, The University of Texas, Austin, 1972].

Department of Mathematics

University of Maryland

Baltimore, Maryland 21228

Department of Mathematics and Center for Numerical Analysis

The University of Texas

Austin, Texas 78712

1. P. M. Anselone \& P. J. Laurent, "A general method for the construction of interpolating or smoothing spline-functions," Numer. Math., v. 12, 1968, pp. 66-82. MR 40 \#3145.

2. G. D. BYRNe \& D. N. H. CHI, "Linear multistep formulas based on $g$-splines," SIAM J. Numer. Anal., v. 9, 1972, pp. 316-324.

3. C. Carasso \& P. J. Laurent, On the Numerical Construction and the Practical Use of Interpolating Spline-Functions, Proc. IFIP Congress Information Processing 68 (Edinburgh, 1968 ), vol. 1, Mathematics, Software, North-Holland, Amsterdam, 1969, pp. 86-89. MR 40 \#8219.

4. P. J. Davis, Interpolation and Approximation, Blaisdell, Waltham, Mass., 1963. MR 28 \#393.

5 C. DE Boor, "On calculating with B-splines," J. Approximation Theory, v. 6, 1972, pp. $50-62$.

6. M. Golomв, Spline Approximations to the Solutions of Two-Point Boundary-Value Problems, MRC 1066, Univ. of Wisconsin, Madison, Wis., 1970.

7. T. N. E. Greville, Introduction to Spline Functions, Proc. Sem. Theory of Applications of Spline Functions (Math. Res. Center, Univ. of Wisconsin, Madison, Wis., 1968), Academic Press, New York, 1969, pp. 1-35. MR 39 \#1868.

8. J. W. JEROME \& L. L. SCHUMAKER, "On Lg-splines," J. Approximation Theory, v. 2, 1969, pp. 29-49. MR 39 \#3201.

9. J. W. Jerome \& L. L. SChumaker, "Local bases and computation of $g$-splines," Methoden und Verfahren der Mathematische Physik, v. 5, 1971, pp. 171-199.

10. S. KARLIN, "Best quadrature formulas and splines," J. Approximation Theory, v. 4, 1971, pp. 59-90. MR 43 \#2403.

11. T. LyCHE \& L. L. Schumaker, "Computation of smoothing and interpolating natural splines via local bases," SIAM J. Numer. Anal. (To appear.)

12. M. J. MunTEanu, Contributions à la Théorie des Fonctions Splines à une et à Plusieurs Variables, Dissertation, Louvain, 1970.

13. I. J. SChOENBERG, "On the Ahlberg-Nilson extension of spline interpolation: the g-splines and their optimal properties," J. Math. Anal. Appl., v. 21, 1968, pp. 207-231. MR 36 \#6849.

14. M. H. Schultz \& R. S. VARgA, "L-splines," Numer. Math., v. 10, 1967, pp. 345-369. MR 37 \#665.

15. L. L. Schumaker, Some Algorithms for the Computation of Interpolating and Approximating Spline Functions, Proc. Sem. Theory and Applications of Spline Functions (Math. Res. Center, Univ. of Wisconsin, Madison, Wis., 1968), Academic Press, New York, 1969, pp. 87-102. MR 39 \#687. 